\title{
Identification of Optimal Path in Power System Network Using Bellman Ford Algorithm
}

\author{
S. Hemalatha ${ }^{1}$ and P. Valsalal ${ }^{2}$ \\ ${ }^{1}$ Department of Electrical \& Electronics, St. Josephs College of Engineering, Chennai 600119, India \\ ${ }^{2}$ Department of Electrical \& Electronics, Anna University, Chennai 600025, India
}

Correspondence should be addressed to S. Hemalatha, latharaju73@gmail.com

Received 25 April 2012; Accepted 9 August 2012

Academic Editor: Dariusz J. Gawin

Copyright (C) 2012 S. Hemalatha and P. Valsalal. This is an open access article distributed under the Creative Commons Attribution License, which permits unrestricted use, distribution, and reproduction in any medium, provided the original work is properly cited.

\begin{abstract}
Power system network can undergo outages during which there may be a partial or total blackout in the system. In that condition, transmission of power through the optimal path is an important problem in the process of reconfiguration of power system components. For a given set of generation, load pair, there could be many possible paths to transmit the power. The optimal path needs to consider the shortest path (minimum losses), capacity of the transmission line, voltage stability, priority of loads, and power balance between the generation and demand. In this paper, the Bellman Ford Algorithm (BFA) is applied to find out the optimal path and also the several alternative paths by considering all the constraints. In order to demonstrate the capability of BFA, it has been applied to a practical $230 \mathrm{kV}$ network. This restorative path search guidance tool is quite efficient in finding the optimal and also the alternate paths for transmitting the power from a generating station to demand.
\end{abstract}

\section{Introduction}

Whenever power interruption takes place it is imperative to bring back the system promptly to its initial state or to an optimal operating network. The problem of obtaining a target network is called power system restoration. It consists of two steps, the first step is to determine an optimal configuration and the second step is to prepare a sequence of switching operations (restoration plan) in order to bring the faulted network into the obtained target system.

Power system restoration is a multiobjective, multivariable, multiconstraint, and mixed optimization problem. It is very difficult to establish mathematical models. The most relevant literatures pertaining to power restorations are discussed. Barsali et al. reported the main results of a comprehensive survey on behalf of an independent system operator [1]. The main strategies, critical issues, and practical experiences related to the restoration plans of a wide set of deregulated systems worldwide are presented. Further, the paper proposes possible innovative enhancements being studied for the intricate sample system, aimed at increasing the effectiveness of the restoration service.
Naggar et al. implemented a relatively new RECRÉ software program (French acronym for "Remise En Charge du Réseau"-System Restoration) by Hydro-Québec in 2005 [2]. The aim of this knowledge management system is to have system restoration knowledge that is tangible, growthoriented, and enhanceable asset. The system is made up of two modules: the knowledge engineering module, whereby modeling can be done of the system restoration strategy and known solutions in a knowledge base, and the planning module, whereby system restoration plans can be drawn up based on the unavailability of power system equipment. The RECRÉ system can produce a validated and adequate plan in a few seconds along with all the documents required for its dissemination.

Nezam et al. reported novel sectionalizing method for the build-up strategy in power system restoration [3]. One of the most practical and economical is the build-up strategy that includes the process of restoring separated parts (islands) in the power system and interconnecting them afterwards are presented. The study intends to develop a systematic algorithm for sectionalizing a power system considering various constraints such as black-start capability of generators, 
power supply-demand balance, and independence of islands. The proposed method is also applied to the IEEE 118 bus system as a large-scale power system to prove its capability in practical systems.

Sun presented a decision support tool, developed in an EPRI project, using Generic Restoration Milestones (GRMs)based strategy employed to provide a quantitative way for evaluating the optimal installation location and amount of BS capability [4]. Based on the proposed criteria, the benefit from additional BS capability is quantified in terms of system restoration time. The IEEE Reliability Test System (RTS) 24bus test system is used for validation of the proposed strategy, using the System Restoration Navigator (SRN) restoration tool. Lin and Wen presented and analyzed several issues governing power system restoration in the traditional power industry and in the electricity market environment [5]. Subsequently, the impacts of the restructuring efforts on the power system restoration are identified and clarified from several aspects including black-start, restoration tests, dispatching, and distribution system service restoration.

Teo and Shen proposed a rule-based approach to generate a dynamic restoration plan in a total or partial black out power system network [6]. Rules are framed for generator, load, and transmission lines. Based on these rules, a dynamic restoration plan is obtained. During this process the restoration system monitors the implementation of the restoration plan by measuring the system frequency, the generator output, voltage at each bus, power factor, and load at each point. The restoration plan is updated if there are deviations from the telemetry data.

After sketching the literature reports it is observed that the application of BFA method for optimal path determination for sequential intricate systems have not been discussed in detail. Hence an attempt is being made in this research to address this issue and propose the systematic methodology to overcome the existing drawback and terminologies not emphasized in the available literature.

1.1. Various Techniques Available for Power System Restoration. Restoration of power in electrical network can be obtained by (i) knowledge-based method and (ii) mathematical programming technique. In earlier days, the knowledge based methods were used, as the time required for restoration is less. For a particular system, based on the forecasting studies, fault analysis, operating philosophies, plant capabilities, expert knowledge, rules are framed. These rules are developed and it is validated under different operating conditions. In the mathematical programming technique the objective function is formulated along with its constraints. Using any one method of optimization or graph theory technique, objective function is solved.

1.1.1. Optimization Method. To solve the problems of restoration using the optimization technique, computer based-systems are very useful. This makes the execution process faster but the major disadvantage is the time required for obtaining the solution for a large complex system is increased.
1.1.2. Graph Theory Technique. Power system restoration can be effectively solved by graph theory technique. The drawback of optimization can be minimized using the technique of graph theory. Application of this method increases the search time as the number of de-energised areas increase. The shortest path finding algorithms are used to find the minimum weighted or most efficient path in the network. In graph theory, it is used to identify a path between two vertices (or nodes) such that the sum of the weights of its constituent edges is minimized. This problem is called the single pair shortest path problem. The shortest paths from all vertices in the graph to a single destination vertex is called single destination shortest path problem. The shortest path between every pair of vertices is called all pairs shortest path problem. Dijkstra's algorithm finds solution in the single-pair, single-source, and single-destination shortest path problem. Johnsons's algorithm identifies the solution in all pairs shortest path problem. The Floyd Warshall algorithm is a graph analysis algorithm for identifying the shortest path between all pairs of vertices is obtained in a single execution of the algorithm. It is an example of dynamic programming. Bellman Ford algorithm obtains solution in the single-source problem if the edge weights are negative too.

\section{Theoretical Background}

2.1. The Description of BFA. The Bellman Ford algorithm was developed by Richard Bellman and Lester Ford Jr. It is a label connecting algorithm which computes single-source shortest route in a weighted graph where some of the edge weights may be negative. Every route in a weighted graph has a corresponding route weight, the cumulative value of which is the sum of the weights of those route edges. The shortest path algorithm is the problem of finding the route between two vertices such that the sum of the weights of its constituent edges is minimized.

In a weighted, direct graph, $G=(V, E)$ with source $S$ and weight function $W=E \rightarrow R$, the algorithm returns a boolean value representing whether or not there is a negative weight cycle that is, reachable from the source. A solution exists if there is negative cycle and it determines the shortest path if there is no such cycle. The algorithm uses relaxation, reducing an estimate $d(v)$ on the weight of a shortest path from the source $S$ to each vertex $v \in V$ until it obtains the actual shortest path weight $\delta(S, v)$. The algorithm returns TRUE if and only when the graph contains no negative weight cycles that are reachable from the source; see Algorithm 1.

The Bellman Ford algorithm is executed in the following steps for the sample 6 bus system shown in Figure 1.

Step 1. Assign infinity to all nodes other than the source. The source node 1 is set as zero as shown in Figure 1.

Step 2. Relax each edge for $(n-1)$ times where $n$ are the number of nodes. So relax edges for 5 times since $n$ is 6.Relaxing an edge means checking to see if the path to the node to which the edge is pointing can be shortened, and if 


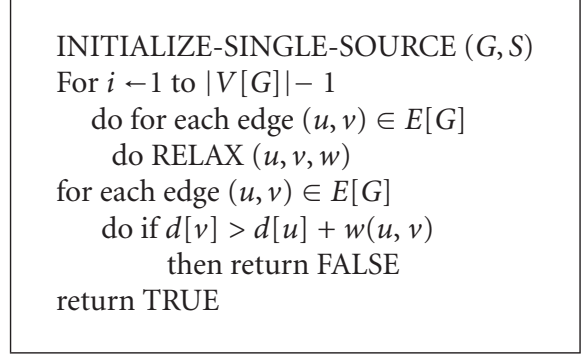

Algorithm 1: Bellman ford $(G, W, S)$.

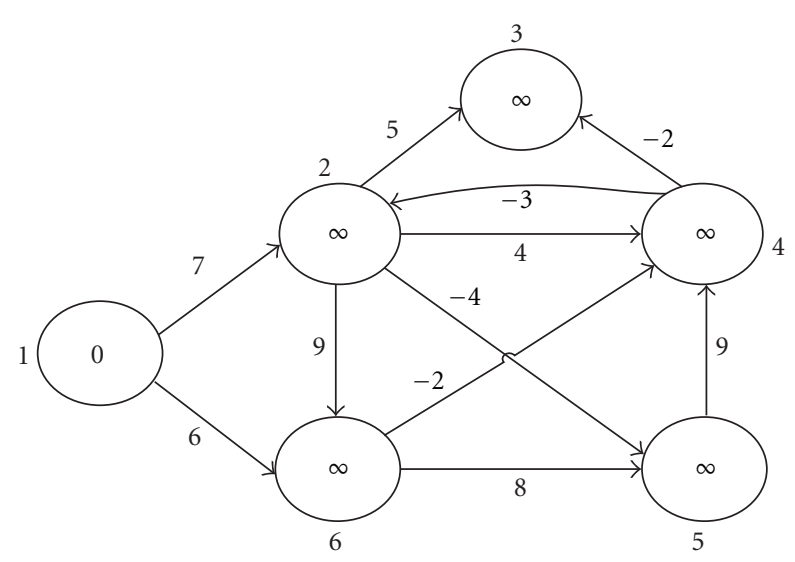

FIgURE 1: Nodal representation for Step 1.

so, replace the path to the node with the found path. Relax the edge with only 2 nodes starting from the source node, consider the edge $1 \rightarrow 2$ of cost 7 , the cost of the source node plus the cost of the edge $1 \rightarrow 2$ is less than infinity means replace the node 2 with the new cost. Similarly relax edge $1 \rightarrow 6$ of cost 6 which is also less than infinity. It is shown in Figure 2.

Step 3. Consider the path with 3 nodes and relax edge $1 \rightarrow 3$ through $1 \rightarrow 2 \rightarrow 3$, relax edge $1 \rightarrow 4$ through $1 \rightarrow 2 \rightarrow 4$ or $1 \rightarrow$ $6 \rightarrow 4$, and relax edge $1 \rightarrow 5$ through $1 \rightarrow 6 \rightarrow 5$ or $1 \rightarrow 2 \rightarrow 5$. The execution of this step is shown in Figure 3.

Step 4. Consider the path with 4 nodes and relax edge $1 \rightarrow 2$ through $1 \rightarrow 6 \rightarrow 4 \rightarrow 2$ and relax edge $1 \rightarrow 3$ through $1 \rightarrow 6 \rightarrow$ $4 \rightarrow 3$. The network is changed as shown in Figure 4 .

Step 5. Consider the path with 5 nodes and relax edge $1 \rightarrow$ 5 through $1 \rightarrow 6 \rightarrow 4 \rightarrow 2 \rightarrow 5$ and relax all edges as shown in Figure 5.

Thus all edges are relaxed and the negative cost cycle is checked and the appropriate boolean value is returned. The final step gives the shortest path between each node and the source node. Hence it is called the single-source shortest path

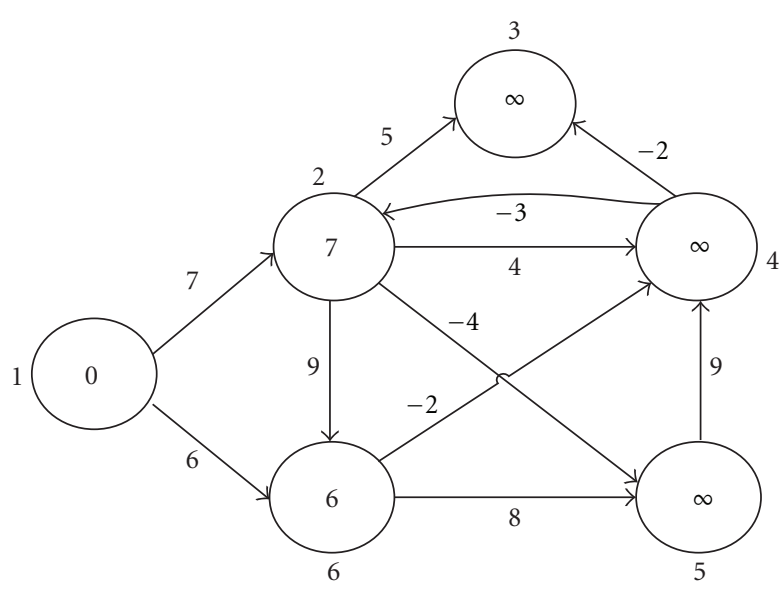

FIGURE 2: Nodal representation for Step 2.

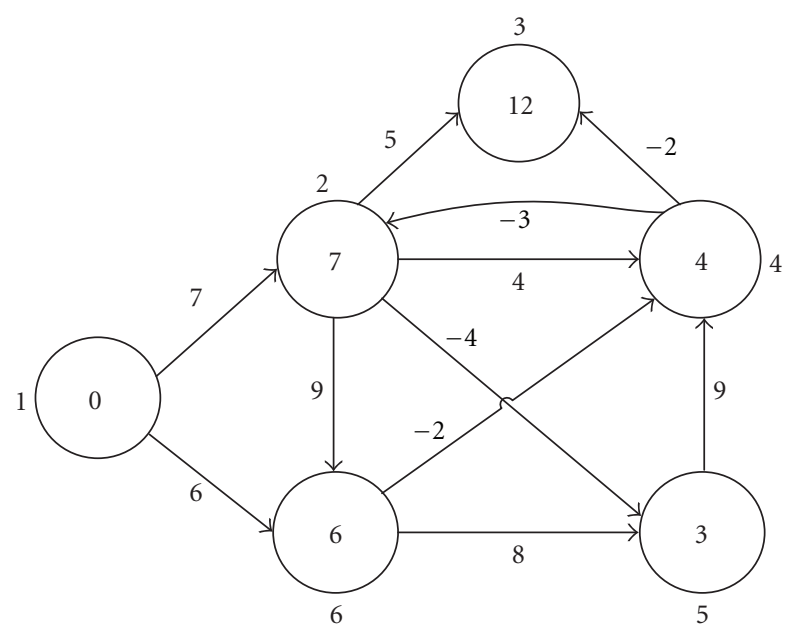

Figure 3: Nodal representation for Step 3.

algorithm. The computational procedure of the BFA for the identification of optimal path is given in Figure 6.

\section{Problem Formulation}

In a bulk power system, there are many generators, tielines, transmission lines, transformers, and loads. If the power system encounters a partial or complete blackout, the network should be reconfigured as soon as possible. In order to restore the network, the power has to be transmitted through the optimal path. This problem is formulated as follows.

Objective Function. The problem of finding the optimal path for restoration can be formulated as a problem of minimizing the cost of each transmission line.

Constraints. Consider the following. 


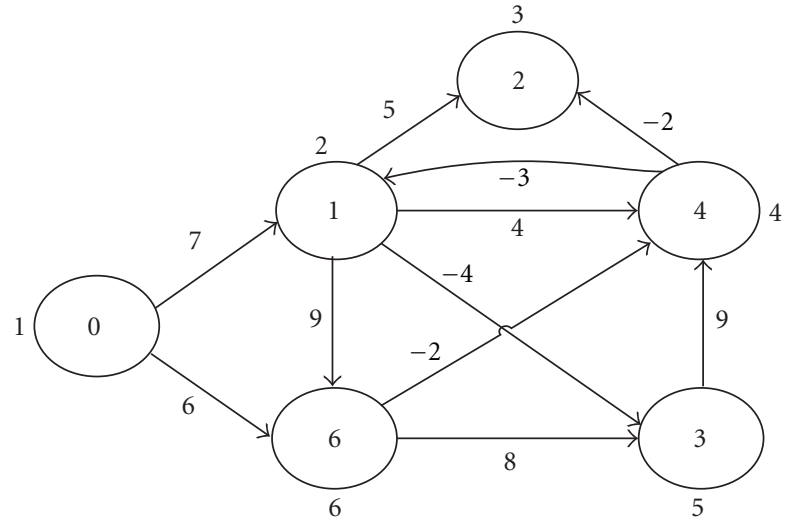

Figure 4: Nodal representation for Step 4.

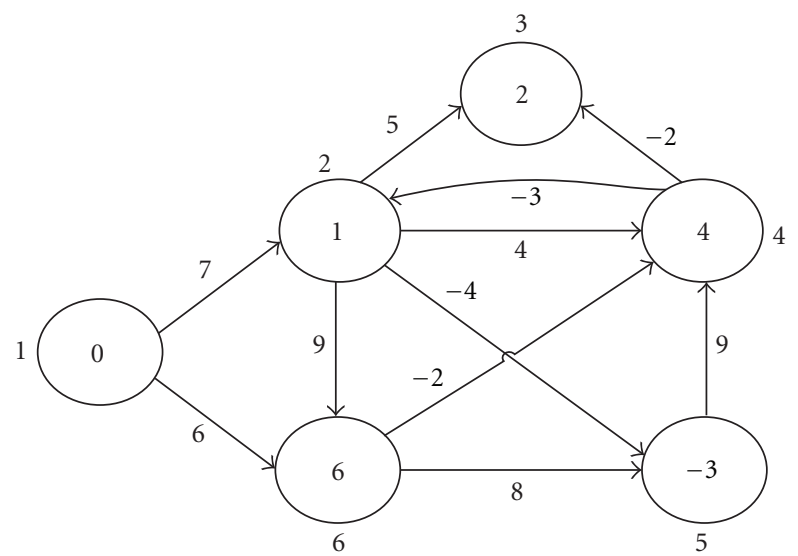

Figure 5: Nodal representation for Step 5.

TABLe 1: Part of the computed results for shortest path.

\begin{tabular}{|c|c|c|c|}
\hline \multicolumn{2}{|c|}{ Node } & \multicolumn{2}{|c|}{ Path } \\
\hline From & To & Optimal path & Other available paths \\
\hline 1 & 10 & $1 \rightarrow 10$ & $\begin{array}{l}1 \rightarrow 5 \rightarrow 10 \\
1 \rightarrow 5 \rightarrow 6 \rightarrow 10\end{array}$ \\
\hline 2 & 17 & $2 \rightarrow 7 \rightarrow 17$ & $\begin{array}{l}2 \rightarrow 5 \rightarrow 10 \rightarrow 7 \rightarrow 17 \\
2 \rightarrow 1 \rightarrow 7 \rightarrow 17\end{array}$ \\
\hline 14 & 16 & $14 \rightarrow 15 \rightarrow 13 \rightarrow 10 \rightarrow 16$ & $\begin{array}{l}14 \rightarrow 15 \rightarrow 17 \rightarrow 16 \\
14 \rightarrow 15 \rightarrow 13 \rightarrow 17 \rightarrow 16\end{array}$ \\
\hline 19 & 17 & $19 \rightarrow 13 \rightarrow 17$ & $\begin{array}{l}19 \rightarrow 13 \rightarrow 10 \rightarrow 7 \rightarrow 17 \\
19 \rightarrow 13 \rightarrow 10 \rightarrow 16 \rightarrow 17\end{array}$ \\
\hline 18 & 6 & $18 \rightarrow 17 \rightarrow 7 \rightarrow 10 \rightarrow 6$ & $\begin{array}{l}18 \rightarrow 17 \rightarrow 16 \rightarrow 10 \rightarrow 6 \\
18 \rightarrow 17 \rightarrow 7 \rightarrow 10 \rightarrow 5 \rightarrow 6\end{array}$ \\
\hline
\end{tabular}

(a) Constraint of the capacity of the transmission line: the power flow in the line has to be below the capacity of the line.

(b) Constraint of distance between source and demand: the distance is calculated based upon the actual impedance of the network. The impedance between the source and the demand for restorative path should be minimum then the losses can be minimized. (c) Constraint of power balance between supply and demand: the available power should be equal to the load demand.

(d) Constraint of voltage stability: the voltage should be within the acceptable limits.

(e) Constraint of priority of load: the important loads are given priority to connect it to the source.

Cost is assigned for each transmission line based upon the constraints and the cumulative cost of the line is calculated. The BFA is executed once to find the path with minimum cost and is identified first. Then it is checked for capacity of the transmission line, power balance between the generation and demand and priority of load and displayed the optimal path. Then the load flow program is conducted to validate the voltage limit. The computational procedure of the optimal path identification is given in Figure 6.

\section{Numerical Results}

In order to validate the algorithm, it has been applied to a practical $230 \mathrm{kV}$ network. The optimal path from generation to demand is identified using the constraints like the shortest path (minimum losses), capacity of the transmission line, priority of loads and power balance between the generation and demand. Based upon the constraints, the cost is assigned for each line and by relaxing the edges, the shortest path with minimum cumulative cost line is selected and is checked for other constraints. Then the optimal path is confirmed by conducting the load flow.

4.1. Implementation of BFA to a Practical $230 \mathrm{kV}$ Network. The Chennai $230 \mathrm{kV}$ Network, a load dispatching centre, provides electricity to more than one million customers in Chennai metropolitan city in India. The network has one black start generator of capacity $120 \mathrm{MW}$ which can be used to crank the other non black start generators. The network is tied with the national grid of India. The national grid (bus 18) supplies $220 \mathrm{MW}$ of power through the Neyveli power station. The network has a total installed capacity of $2300 \mathrm{MW}$ and it is shown in Figure 7.

The computer program is written in JAVA using JDK1.5 to display the optimal path for a particular source and demand. If there is any fault in the network, load extension, or maintenance work the network topology will be changed. At that time the line can be added or deleted in the software by altering the cost of the transmission line. Consider, the power has to be transmitted from the generating station MAPS, bus number 19, to the area koyembedu, bus number 6. The algorithm interspaces all other nodes in between 19 and 6 and the paths are checked for other constraints then the available paths are displayed in which the first path is the optimal path. Because of the unavailability of the operator in the substation, the failure in the equipment, the first path cannot be executed means the other alternate paths can be considered and it is shown in Figure 8.

The shortest paths for all possible pair of nodes are found out and a few of them are shown in Table 1. Based on this 


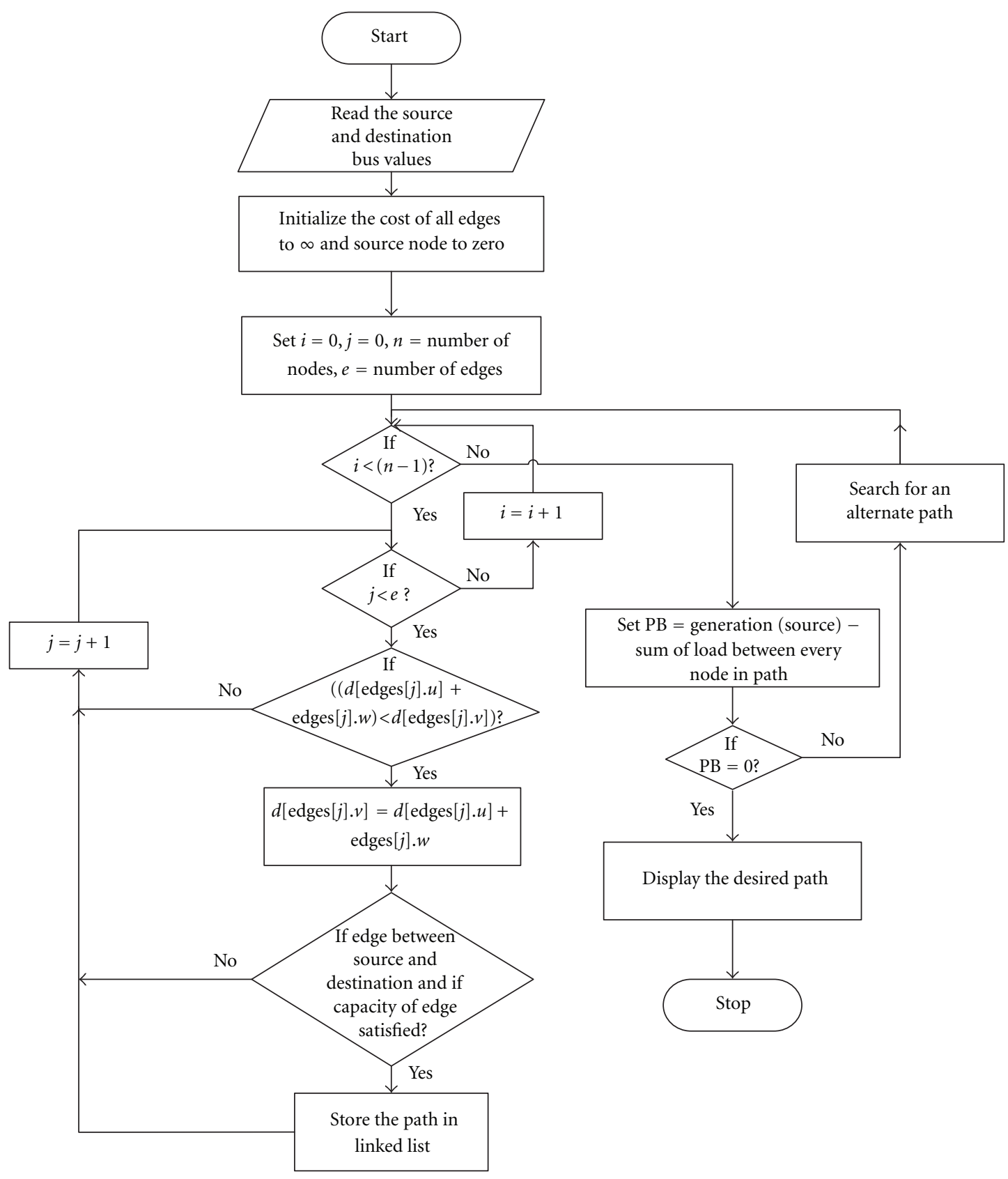

FIGURE 6: Computational procedure for optimal path identification.

guidance, the power system operators can transmit the power through the line without any stress. So that the execution of restoration plans will be efficient.

\section{Conclusion}

Identification of optimal path for transmission of power is the major difficulty in the area of power system restoration. But it is very essential to restore the network in minimum time. Many proposed shortest path-finding algorithms tackle this problem in the area of transportation and computer networks by transforming the problem into the shortest path selection problem. But in the case of power system restoration, selecting the shortest path is not a hard problem, but the shortest path is not always the optimal path. So for a practical network in dynamical environment the restorative power flow paths are to be specified according to variable constraints. BFA is applied to find out the optimal path and also the several alternative paths by considering not only the shortest path (minimum losses), but also the capacity of the transmission line, voltage stability, priority of loads, and power balance between the generation and demand. A systematic guidance tool is developed, which reduces the 


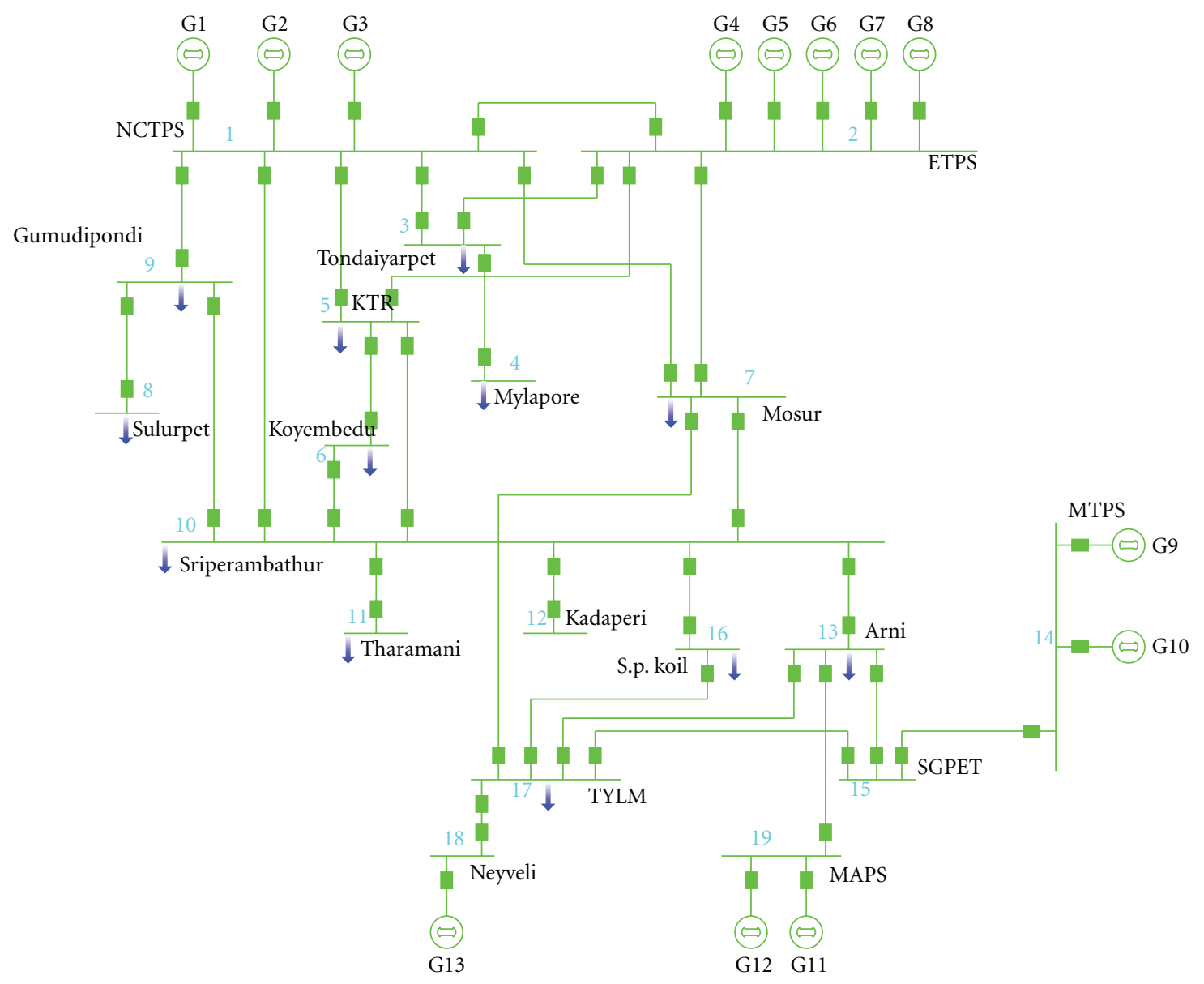

FIgURE 7: Chennai $230 \mathrm{kV}$ network.

\begin{tabular}{|c|c|}
\hline ci Command Prompt - java Search & $-\square x$ \\
\hline 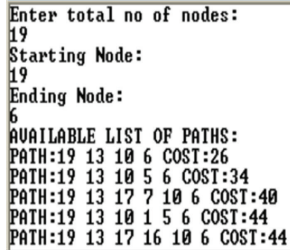 & \\
\hline
\end{tabular}

FIgURE 8: Results of Chennai $230 \mathrm{kV}$ network.

possibility of error operation caused by the power system operator and makes the restoration safer and faster. In order to validate the algorithm, it has been applied to a practical $230 \mathrm{kV}$ network. From the obtained results it is found that the optimal path provides better satisfaction to the power system operator with minimum operating time and reduces the stress of the operator.

\section{References}

[1] S. Barsali, R. Giglioli, D. Poli, M. Sforna, R. Salvati, and R. Zaottini, "The restoration of an electric power system: International survey and discussion of possible innovative enhancements for the Italian system," Electric Power Systems Research, vol. 78, no. 2, pp. 239-247, 2008.

[2] R. Naggar, L. Cauchon, S. Hénault, and S. T. Phan, “The RECRÉ system: restoration planning for Hydro-Québec's power system," in Proceedings of the IEEE Power Engineering Society General Meeting (PES'6), Quebec, Canada, June 2006.

[3] S. A. Nezam, A. S. Dobakhshari, S. Azizi, and A. M. Ranjbar, "A sectionalizing method in power system restoration based on WAMS," IEEE Transactions on Smart Grid, vol. 2, no. 1, pp. 178185, 2011.

[4] W. Sun, "Black start capability assessment in power system restoration," in Proceedings of the IEEE Power and Energy Society General Meeting (PES'11), pp. 1-7, San Diego, Calif, USA, 2011.

[5] Z. Lin and F. Wen, "Power system restoration in restructured power industry," in Proceedings of the IEEE Power Engineering Society General Meeting (PES’07), June 2007.

[6] C. Y. Teo and W. Shen, "Development of an interactive rule-based system for bulk power system restoration," IEEE Transactions on Power Systems, vol. 15, no. 2, pp. 646-653, 2000. 

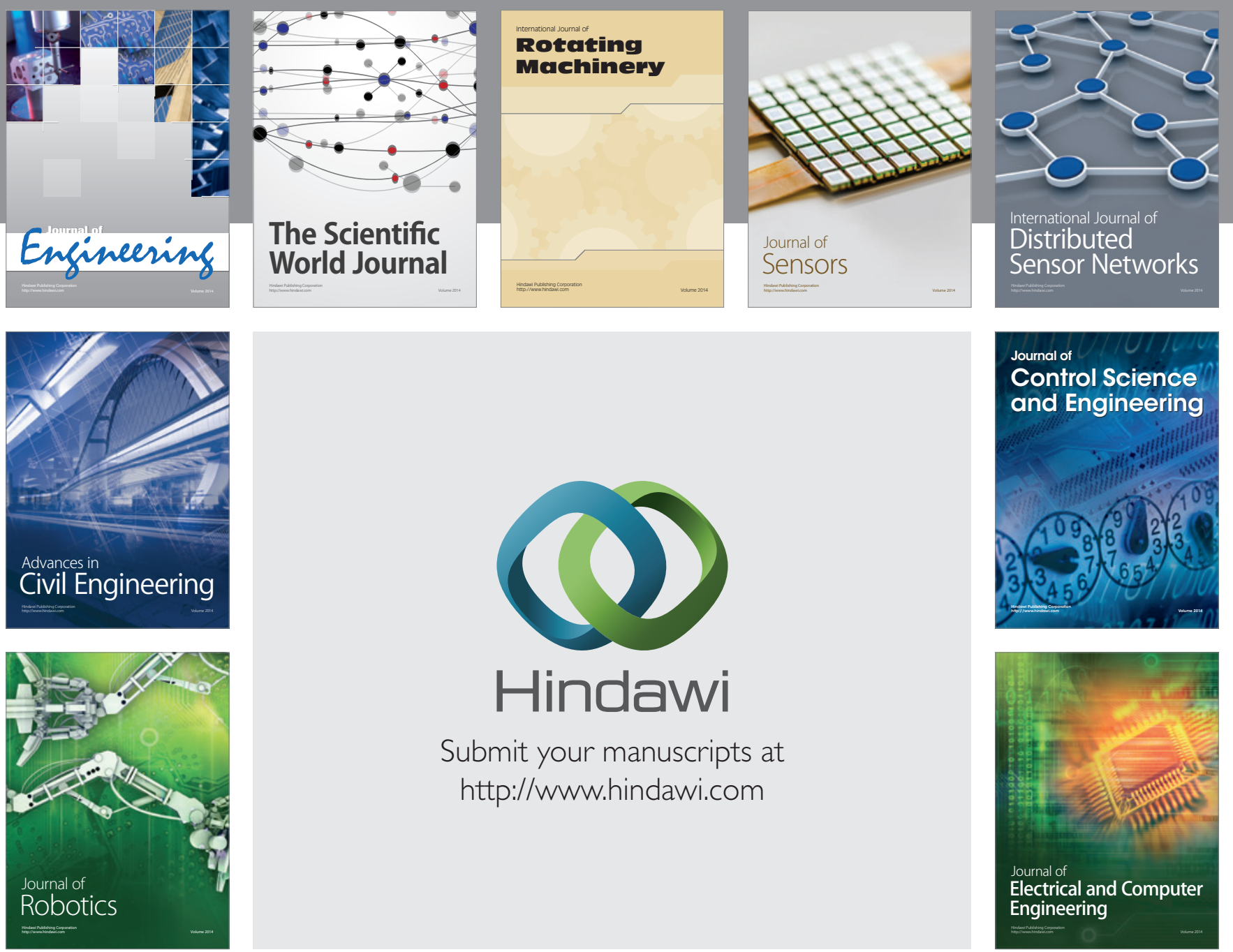

Submit your manuscripts at

http://www.hindawi.com
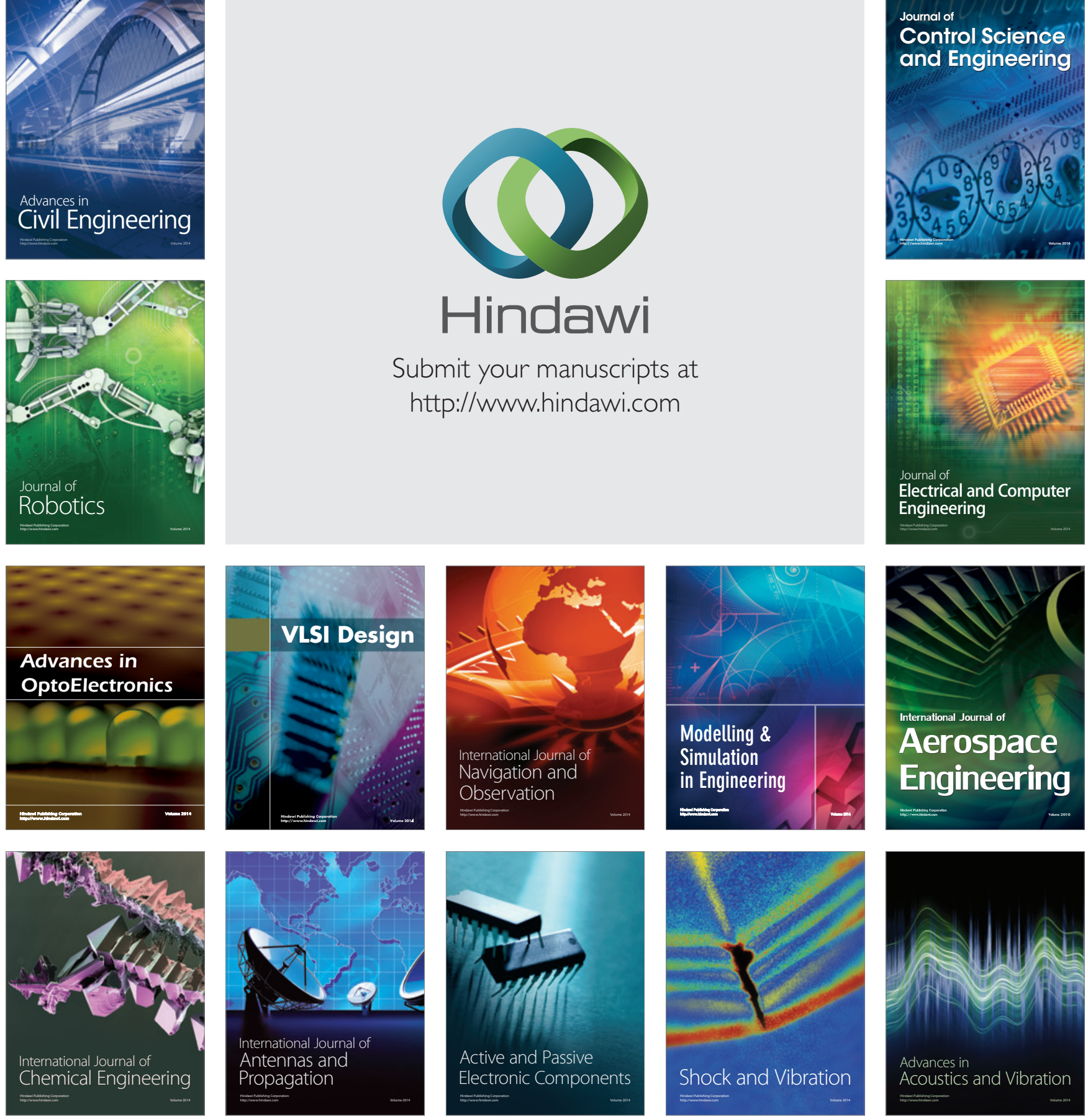